\title{
Les techniques dramatiques dans le Square de Marguerite Duras
}

\section{Ahmed Moawad Abd-Elhadi*}

ahmedfilsdunil@gmail.com

\section{Résumé}

Notre travail porte sur une œuvre romanesque de Margueritte Duras. Le Square, c'est un roman théâtral composé uniquement de dialogues et monologues entre deux êtres à l'aide ou à travers des didascalies, on l'imagine joué dans un théâtre : Tous ces éléments théâtraux quand ils existent en dedans du roman sont pour tout le monde un roman théâtral. Notre objectif a porté sur les techniques caractérisant l'écriture de Duras dans le Square, et en même temps, nous avons dégagé la théâtralité. De là, on a pu mettre en évidence certains procédés de théâtre dans ce roman : dialogue, monologue, didascalies. Etc. Tous ces éléments ont formé en définitive l'essentiel du Square.

Mots-clefs : Dialogue - didascalie - Margueritte Duras monologue - roman théâtral

* Professeur Adjoint de littérature française, Faculté des Lettres, Université d'El-Arich 


\section{Introduction}

La littérature féminine d'expression française occupe « une place de choix dans le domaine des lettres françaises à l'étranger, à côté des littératures canadiennes, africaines ou libanaises. " ${ }^{(1)}$ Les femmes-auteurs sont encore plus rares. L'une d'entre elles a commencé, en dernière période du XXe siècle, une carrière d'auteur : Mme Margueritte Duras qui occupe une grande place dans la littérature française par son œuvre romanesque et théâtrale. Elle a connu un succès éclatant aussi bien en France qu'à l'Etranger dans la dernière période du XXe siècle. Elle est célèbre par une étude approfondie au niveau de l'écriture. Duras est aussi scénariste et membre du Jury du Festival de Cannes et du prix Femina. Elle est classée parmi les grandes figures du nouveau roman du siècle dernier. Son œuvre est marquée par la contamination des genres littéraires, c'est-à-dire on y trouve un mélange du roman au théâtre.

Elle est sans doute l'une des écrivains français les plus actuels. Elle jouit d'une large réputation européenne grâce à sa grande production littéraire.

Dans le Square, Marguerite Duras raconte la vie de gens modestes surtout les marginaux. Une discussion s'engage entre un homme et une femme dans un square parisien. Les deux personnages, se trouvant à la marge de la société, se posent la question sur la place qu'ils y occupent.

D'abord, l'écriture de Marguerite Duras nous assure qu'elle est capable d'écrire des scènes dramatiques et une véritable mise en scène. C'est le cas du Square (2). Nous pouvons dire que c'est un roman dialogué ou plutôt une pièce de théâtre. Ce sont les deux à la fois. À ce stade, voyons-nous que le Square est un texte film théâtre, qui se compose de trois actes distincts. Mais ceux-ci ont lieu à la même place, 
seulement l'action est changée. Non seulement, ce livre a la structure d'un roman au niveau de la mise en page, mais aussi à la structure d'un scénario, d'un texte, d'une pièce de théâtre. A ce propos, Duras a une attitude en matière théâtrale : "Elle croit moins à la nécessité de jouer qu'à celle de lire, à voix haute dans le bon tempo, avec une intonation juste " ${ }^{(3)}$ elle voit que le roman devrait être lu, non pas joué.

L'œuvre de Margueritte Duras fait partie de la littérature ethnographique. Nous pouvons dire qu'elle est une écrivaine ethnographique parce qu'elle, incarne dans ses textes, la société franco-vietnamienne avec ses multiples facettes. Non seulement, elle décrit les couches de la société, mais aussi, elle met une interrogation sur les valeurs du monde.

Ce travail tend à mettre en lumière l'importance des lettres françaises au Viêt-Nam, à porter ensuite un jugement de valeur sur l'écrivaine et ses œuvres le plus marquantes, "le goût actuel pour l'autobiographie, les recherches de la critique universaire sur ce que l'on appelle "l'écriture du moi», ou "écriture de soi », montrent la force de cet intérêt. » ${ }^{(4)}$

La recherche nous a révélé une tentation de découvrir la spécificité et l'originalité de l'écriture chez Duras dans le square; elle nous a permis d'appréhender certains aspects essentiels dans ce roman. La forme du roman est assez étrange. Il est fait d'un long dialogue interrompu uniquement par quelques phrases courtes expliquant ce qui se passe dans l'environnement des protagonistes, ce qui introduit des respirations bienvenues tout autant que nécessaires.

\section{La division du roman en acte}

Il s'agit d'un roman de 150 pages publié en 1955, soit relativement tôt dans la carrière de l'écrivaine. Il a pour caractéristique d'être fait en forme dialogue. Ce roman est divisé en trois actes. L'action se passe dans un square, sur un banc. Tout 
le livre n'est qu'on long dialogue entre deux personnes qui se sont rencontrés par hasard, dans ce square. La singularité du plus récent roman de Marguerite Duras et, pareillement, de sa version théâtrale, se base sur nombreux éléments tels que les actes théâtraux comme nous voyons dans la structure du roman qui comprend trois actes suivants.

\section{Acte I}

L'héroïne est identifiée avec une narratrice qui, bien qu'elle cache son nom pendant un temps. Il y a d'une part une jeune femme dont le travail est de s'occuper de l'enfant d'une autre, et de l'autre un représentant de commerce plus âgé. Beaucoup de choses les opposent, mais ils vont se rapprocher naturellement grâce à un horizon qui pourrait être commun. En effet, la fille est jeune, elle a foi en l'avenir mais se trouve bloquée par toutes les contraintes qu'elle rencontre dans la vie.

\section{Acte II}

Elle veut changer de métier, de vie, elle veut vivre différemment et elle est prête à tout sacrifier pour arriver à ses objectifs même à sa liberté. Elle cherche par tous les moyens à trouver un mari qui la rassurera et la débarrassera du poids de son quotidien. Pour parvenir à ses fins elle se rend tous les samedis au bal où elle espère rencontrer l'homme providentiel.

\section{Acte III}

A l'opposé l'homme, lui, est blasé. Il n'a pas de racine, il se déplace tout le temps, et a vu dans sa vie trop de malheur pour croire en l'avenir. Il ne croit pas dans le couple, dans l'avenir à deux. Pourtant, au fil de la conversation, ils vont affronter leurs points de vue jusqu'à découvrir qu'ils ont peut-être un avenir ensemble. Le roman se termine dans l'espoir que met la fille dans la venue au bal de l'homme, au prochain samedi. Il est évident que la venue de la fille au bal de cet homme à la fin du récit est marquée par la retombée d'un rideau. 


\section{Le fantasme :}

La notion réfère à "l'action imaginaire qui implique le sujet, et figure, au travers de processus défensifs, l'accomplissement d'un désir (inconscient). » ${ }^{5}$ Avec un titre pareil nous pouvons tout imaginer et c'est bien là où réside la première force de cette œuvre : avant même de lire les premières pages, le lecteur se voit déjà s'engouffrer dans un thriller démoniaque ou dans une histoire d'amour victorienne selon ses références. On se demande quelle farce elle a encore inventé "La Duras" : s'agit-il d'un roman vraiment ? Ou plutôt d'une pièce de théâtre ? Probablement une forme hybride, une forme à la manière de Duras, qui n'en finit pas de creuser la création littéraire. Une œuvre de jeunesse publiée en 1955. Alors non dans ce square rien ne se passera : ni meurtre, ni grande déclaration d'amour. Juste ce qu'il s'y passe, "dans la vraie vie, lorsque se croisent les gens "de peu", les presque invisibles, ceux qui font "trois petits tours et puis s'en vont' dans la fuite du temps. » ${ }^{(6)}$

Le fantasme se base sur la scène qui se passe dans un jardin, sur un banc. Tout l'ouvrage n'est fantastiquement qu'on long dialogue entre deux personnes qui se sont rencontrés par hasard, dans ce square. Il y a d'une part une jeune femme dont le tâche est de s'occuper de l'enfant d'une autre, et un représentant de commerce plus âgé.

Pour parvenir à ses fins, elle se rend tous les samedis au bal où elle espère rencontrer l'homme heureux. La scène du bal joue un rôle très important dans le roman. L'écrivaine fait du bal un moment capital de l'action cristallisant les plus grands espoirs et les plus grands drames de l'amour. Cette scène qui ouvre le roman par la jeune fille de vingt ans qui est domestique à tout faire et espère se marier, l'homme n'a pas de domicile et il voyage sans cesse pour vendre ses articles sur les marchés. Tous deux ont des attentes et une conception différente de la vie, mais tous deux 
sont seuls et ont envie de parler. Les voilà réunis pour un certain temps dans un square où la jeune fille emporte l'enfant qu'elle garde, et ils se sympathisent. Dans un style simple voire naï, ils parlent de choses et des autres, et proposent de vraies réflexions philosophiques sur le sens de la vie, les buts à poursuivre, le changement, les relations sociales, le bonheur... Les personnages sont tantôt touchants tantôt irritants, à la fois curieux et timides, posant des questions et avançant des arguments mais ne cessant de s'excuser de le faire.

«-Je vous comprends, Mademoiselle. Moi, voyez-vous, je
viens de faire une assez longue tournée et je me repose. En
général je n'aime pas beaucoup penser à l'avenir et,
aujourd'hui que je me repose, moins encore; c'est pourquoi
j’ai du mal vous expliquer comment je me supportais ainsi, à
ne pas changer, et même à ne pas le prévoir. Excusez-moi.
- C'est moi qui m'excuse, Monsieur.
- Mais non, Mademoiselle, on peut toujours causer.» ${ }^{(7) .}$

Contrairement aux sentiments de l'homme, lui, est dégoûté. Il n'a pas de racine et il a passé dans sa vie par des expériences fâcheuses. Le roman se termine dans l'espoir que met la fille dans la venue au bal de l'homme, au prochain samedi. A ce propos, Borgomano montre comment la scène du bal donne lieu à une véritable «déconstruction » des stéréotypes de l'amour fou et de la femme fatale. ( 8 ')

Il est à noter qu'il y a une analogie entre le bal de ce roman et celui du Bal du Comte d'Orgel. Dans le dernier roman, Raymond Radiguet met en scène la rencontre inattendue et le coup de foudre fatal des amants. Dans notre roman, Un homme dans la quarantaine et une jeune fille de vingt ans se croisent dans un square. Elle est une petite bonne exploitée, qui vient au square avec l'enfant de la famille. Il est un représentant de commerce qui gagne difficilement sa vie. Il n'a plus vraiment d'espoir de changer 
sa condition, se contente de vivoter au quotidien, en espérant quelques moments de bonheur passager. Elle attend de sortir de sa situation en se mariant, et fréquente pour cela régulièrement un bal. La conversation s'engage, chacun tente de comprendre l'autre, et se dévoile progressivement. Une sympathie naît d'emblée, une complicité s'ébauche. Deux vies sans grand éclat se croisent, et quelque part s'éclairent mutuellement, un bref instant, peut-être un peu plus longtemps, mais cela on ne le saura pas.

Cette scène est d'autant plus frappante que le bal se déroule d'après un scénario identique à celui de la scène fameuse de la rencontre de Mahaut et François de Séryeuse, qui ne se connaissent pas encore. Le début des deux scènes est tout à fait significatif parce que le bal s'ouvre dans les deux romans de la même manière sur l'entrée marquée d'un personnage inattendu. Dans Le Bal du Comte d'Orgel, nous pouvons lire :

«De temps en temps, quand il sentait qu'elle (Mahaut) ne pouvait le surprendre, François jetait un coup d'œil sur Mme d'Orgel. Il la trouvait belle, méprisante et distraite.

[ ...] Vivre un conte de fées n'étonne pas. Son souvenir seul nous en fait découvrir le merveilleux. François appréciait mal ce qu'avait de romanesque sa rencontre avec les Orgel. [ ...] La musique joua. François de Séryeuse fut heureux de ce bruit qui lui permettait de se taire. Il se tourna vers Mme d'Orgel, sans penser qu'il lui souriait.» (9)

Le fantasme est la folle du logis, il s'agit de folle se plaisant à dérégler la raison humaine. C'est elle qui vit un conte de fées n'étonne pas, transforme le souvenir seul nous en faisant découvrir le merveilleux. C'est à cette occasion que François rencontre la femme du comte, Mahaut d'Orgel, dont il tombe immédiatement amoureux. Ne s'avouant pas ce sentiment et voulant faire bonne figure auprès d'Anne qu'il apprécie beaucoup 
en tant qu'ami, il est dès lors de toutes les activités des Orgel, devenant rapidement un intime de la maison. Et dans le Square :

«- Je vous demande pardon, monsieur, mais moi, qu'il y ait des cas particuliers, je ne veux pas le savoir. Je vous le répète, j'ai de l'espoir. [ ...] et je danse avec qui m'y invite.et comme on dit que la vérité finit toujours par se reconnaitre, je crois qu'on finira bien un jour par me reconnaitre comme une jeune fille apte à se marier, tout comme les autres.

- Il ne suffirait pas que j'aille au bal pour ma part, vous comprenez, et même si je désirais changer, et de façon moins radicale que vous, mademoiselle. » (10)

Mais cette folle du logis éloigne l'homme du savoir où la vérité finit toujours par se reconnaitre. L'image dans sa profusion est plutôt un leurre de fiction, qui fait retour au même. Nous pouvons également remarquer que chez Margueritte Duras, le côté théâtral et spectaculaire de l'arrivée de l'homme est accentué. En effet, son apparition n'est pas annoncée, comme dans le Bal du Comte d'Orgel, par un bruit, mais au contraire par un grand silence. L'aspect du roman est assez étrange. Il est fait d'un long dialogue cassé seulement par quelques expressions courtes développant ce qui se passe dans l'environnement des protagonistes, ce qui introduit des respirations bienvenues tout autant que nécessaires. L'action se réduit à des paroles, le lecteur "entre donc dans l'intimité des personnages lorsqu'ils se dévoilent l'un à l'autre. Et l'on voit la femme avec un regard très dur sur sa condition. Elle estime que son métier est difficile, elle se pense humble, abandonné. " ${ }^{(11)}$ Elle estime que sa condition est inférieure à celle de l'homme, qui ne la punit pas. Il faut exprimer que lui croit encore pouvoir faire quelque chose dans le prochain, ce que ne croit pas la femme. Le Square, c'est texte riche, plein d'humanité. Deux personnages se dialoguent, dans le respect, 
même si parfois d'une façon théâtrale où la notion du spectacle s'impose surtout par l'accent mis sur la vision; les verbes « voir et regarder » sont répétés avec insistance :

«Parfois l'envie me prend d'y faire un tour mais, seule, voyez-vous, une jeune fille de mon état ne peut pas se le permettre.

- J'oubliais : parfois quelqu'un vous regarde.

- Je vois. Et s'approche, oui.

Sans raison?

C'est une forme d'acceptations qui se finit par sembler que tout est possible et qui est merveilleusement écrite, sans doute pas dans un langage que le type de personnage parlerait en réalité, mais peu importe, nous sommes au théâtre. Une très bonne pièce. C'est ainsi que le drame se dénoue car la fascination " $n$ 'a pas été épuisée. Cette fois, le cours du temps se bloque, se cristallise sur cet instant qui maintient inachevé le spectacle du désir laisse la femme en proie au désespoir de ne pouvoir empêcher cette séparation d'avec elle-même. » (13)

L'écriture est circulaire, cette fois-ci, il s'agit d'un échange entre un homme et une jeune femme dans un square. Il est question d'attentes, de bonheur, de désespoir, de ce qui fait la trame de leurs jours. Pas de suspense, juste deux êtres qui se rencontrent dans leurs différences. Peut-on vivre sans l'envie que des choses changent ? Cela paraît inconcevable pour celle qui veut se marier, pour cesser un travail toujours plus harassant, ne plus être seule. Pire, ne plus être seule et invisible :

..., monsieur, car si peu que vous soyez, vous êtes quand même à votre façon, donc vous ne pouvez pas savoir ce que c'est que n'être rien (14)

Elle lui parle de l'attente de la rencontre d'un homme dans un bal, seule rencontre qui changerait sa vie. Elle voudrait que tout change pour elle, mais ne refuse jamais le travail 
supplémentaire qu'on lui confie, lui au contraire n'a pas besoin de changement et se trouve bien finalement dans sa situation. Chaque jour dans une ville nouvelle.

Il y a toujours un lieu où tout se passe. Le lieu c'est le square. D'autre fois, ce sont des halls, des villas, des hôtels ou des salles, Mais là c'est un square et pour tout dire, pas un jardin, ou pas vraiment. C'est un banc. Le square c'est la page. Le banc c'est leur portée. Et ce que vont se dire ces deux personnes là sur le banc ce sont ses notes. Certains à les voir, comme ça, diraient qu'il ne se passe rien, rien de ce qui pourrait sortir de l'ordinaire. Rien qui mériterait qu'on s'y arrête. Non, mais c'est justement là le point de départ: l"ordinaire. Ça regarde tout le monde et ça ne voit personne. Le quotidien qui doit répondre à ce que vous demande la vie. L'ordinaire de leur réponse. L'extraordinaire c'est d'y penser. Et puis d'écrire sur ça. Mais pour ça il faut que « l'écriture arrête le temps pour qu'on puisse arrêter les gens, et que les mots n'aient plus peur de se poser. Et qu'ils se posent sur un banc et se mettent à parler. Comme ça sans manière, sans prendre la pose. Sans même y songer. " ${ }^{(15)}$ Tout se passe comme s'ils se laissaient aller. Aller de soi vers l'autre et de l'autre en soi. Faut peut-être du courage pour ça. Parler de quoi, Monsieur ? D'espoir, Mademoiselle. Oui, d'espoir Monsieur ? Comment faire autrement Mademoiselle ? Ça fait souffrir, Monsieur. Il faut bien manger, Mademoiselle. Le moment où il apparaît, où il disparaît. Comment est-on avant, après l'espoir. Que devient-on ? L'espoir dans l'encre de Duras c'est un désir qui fait des ronds dans l'eau. Ils pleurent toujours un jour un peu dans les allées du square. Juste une ondée, de quoi éclaircir un peu nos idées, au détour des mots voir apparaître le profil du songe. L'espoir de se revoir. La géométrie des corps forme la musique des mots. Deux notes dans le square se parlaient d'amour vers le soir. Et dire que certains pensent encore qu'il ne se passe rien au square, ça n'existe pas des 
gens sans histoire, ça n'existe pas, comme ça n'existe pas une vie sans espoir.

Un square parisien est le seul endroit où des personnes de classe modeste, des deux genres, se sentent légitimées à se parler. Le dialogue entre un voyageur de commerce - en réalité un colporteur - et une jeune bonne fait toute la matière de ce livre, qui est scandé par l'apparition du petit garçon de cinq ans dont elle a la charge. Le dialogue, avec ses flux et reflux, ressemble aux ondes qui mouillent un rivage... rien ne se produit, la conversation et la compréhension mutuelle avancent et reculent alternativement, parce qu'elles sont fondées sur le non-dit.

Ainsi, s'agit-il d'un texte qui, du théâtre contemporain, possède à la fois l'ellipse entre les reparties, comme si le malentendu était toujours aux aguets, et l'immobilisme qui suggère que la véritable communication est ailleurs que dans le verbal : prétexte ou vrai besoin, peu importe. L'action aussi, sans être précisément niée, est suspendue dans le final ouvert.

\section{Le dialogue}

Le dialogue qui est un de seuils du roman dialogué et du théâtre s'engage dans un square entre un voyageur de commerce solitaire, qui ne se projette pas dans l'avenir, aspire au repos, et une jeune fille qui exerce le métier de bonne à tout faire, qui espère en un futur meilleur. En effet, Le Square est considéré comme un dialogue présenté sous toutes ses formes selon La définition de Boblet qui porte sur la forme du texte dans la mesure où elle définit le roman dialogué comme "un texte de matière romanesque envahi par le dialogue au point que la proportion entre le narratif et le discursif bascule au profit exclusif $d u$ discours » ${ }^{(16}$ ' Ce texte brouille les frontières des genres et crée une zone de turbulence. Corvin montre que le dialogue représente "le mode d'expression dramatique par excellence. " (17) En 
supprimant le narrateur, il explore les voies et les impasses de la communication.

Il y a certains dialogues échangés entre les personnages : entre la femme et son futur de l'homme. Lorsqu'ils font connaissance, la première conversation donne un premier exemple de glissement de la conversation vers le dialogue commençant d'une manière courtoise d'accompagnement puis de renseignement :

«L'enfant arriva tranquillement du fond du square et se planta devant la jeune fille. "J'ai faim ", déclara-t-il.

Ce fut pour l'homme l'occasion d'engager la conversation.

"C'est vrai que c'est l'heure de gouter ", dit-il.

La jeune fille ne se formalisa pas. Au contraire, elle lui adressa un sourire de sympathie.

"Je crois, en effet, qu'il ne doit pas être loin de quatre heures et demie...» $(18)$

Pouvons dire que les choix dialogués de Duras peuvent donner une résonance très particulière aux fictions des personnages. L'histoire de ce roman est présentée sous forme d'une succession de répliques dialoguées. Le lecteur y passe directement au début à la scène théâtrale, car L'homme va profiter de la présence de l'enfant qu'elle garde pour commencer le dialogue. Le dialogue est la trame du récit. Nous savons qu'elle est "bonne" puisqu'elle garde cet enfant et fait des tâches ménagères et que, lui, il vend des objets dans sa valise sur les marchés. La femme lui conseille que ce n'est pas un métier que le mien. C'est une sorte d'état, comme par exemple d'être un enfant ou 'être malade et elle lui demande : 
« Alors cela doit cesser.

-Je vous comprends, Mademoiselle. Moi, voyez-vous, je viens de faire une assez longue tournée et je me repose. En générale je n'aime pas beaucoup penser à l'avenir $[$...] c'est pourquoi j'ai dû mal vous expliquer comment je me supportais ainsi, à ne pas changer, et même à ne pas le prévoir. "

Considérons que le premier élément caractéristique du roman dialogué est le recours systématique à une scénographie conversationnelle. En effet, le roman dialogué met en scène une grande diversité d'interactions verbales qui ont toutes en commun d'être des mutations de conversations. Prenons d'abord le cas du voyageur. Le lecteur entre dans le roman par une courte introduction composée d'une série de questions portant sur l'identité, l'origine, la destination, l'occupation de deux personnages. Les promenades de jeune fille et l'homme au hasard d'un voyage sont l'occasion d'un échange portant sur la doctrine déterministe, défendue par le héros. Suivre les dialogues devient donc une sorte de défi, entre digressions, récits enchâssés et changements de sujets. En effet, jouant sur les conventions romanesques, Duras recourt à la parodie pour explorer différents registres et genres littéraires. À cet égard, le roman déborde l'appellation picaresque puisqu'il met en jeu, dans les possibilités de la conversation, une réflexion critique à la fois littéraire et philosophique. C'est un dialogue entre les deux personnages : elle lui demande de changer son métier mais il répond toujours qu'il veut se reposer. La dernière phrase exprime une didascalie gestuelle.

Le lecteur retrouve chez Duras, la retombée des mots et la suspension des mots surtout dans des récits d'essai d'amour. A l'intérieur de la phrase, l'inachèvement renvoie à une impossible retombée des mots. Ces inachèvements « correspondent à un effet 
volontaire d'indicible. » cet inachèvement apparait sans doute dans les dialogues. C'est le cas lors des retrouvailles de la jeune fille avec ce voyageur :

« - mademoiselle, je voudrais vous raconter comment je suis rentré dans cette ville après avoir déposé ma valise dans la chambre.

-Oui, Monsieur, mais il ne faut pas vous inquiéter pour moi. Cela m'étonnerait que je me laisse aller à perdre patience un jour ....

-Mademoiselle, ce n'est que le soir, après avoir déposé ma petite valise... ${ }^{(20)}$

Le dialogue est ici un groupe de spectacles, de performance éphémère dans un cadre dialogué devant des lecteurs regardant, un travail écrit, un exercice gestuel et vocal adressés, le plus souvent dans un lieu particulier. Remarquons que la phrase de ce voyageur est suspendue. Puis elle fait détourner le discours de sociabilité vers ce qui le menace. A travers ces dialogues, on rencontre également des autres cas qui expriment matériellement le trouble et la douleur des locuteurs par les points de suspension.

En plus de ces dialogues, pouvons-nous parler de l'interrogation à travers le dialogue. Nous rencontrons des questions émanant au discours rapporté directement de l'un des personnages. Les personnages utilisent beaucoup de propositions incises, quand ils se confrontent face à face. En posant la question sur la durée au son travail, elle ose s'adresser à lui ainsi:

«Monsieur, est-ce que vous pensez que cela va durer pour vous de voyager comme ça? Croyez-vous que vous vous arrêterez un jour?

-Je ne sais pas.

-On cause, n'est-ce pas, Monsieur. Excusez-moi encore de vous poser ces questions. » 
Duras a recours à cette méthode pour donner au lecteur les indications essentielles et suffisantes pour attribuer chaque question à son personnage à des moments de doute. L'écrivaine utilise une proposition introductrice pour faire entrer le narrateur dans l'interrogation à la place de la jeune fille : «Je demande » Celle-ci emploie le tutoiement envers l'homme opposé au vouvoiement du héros qui veut retourner au récit.

La figure de conversation dans la scène nous conduisant alors à une reconnaissance argumentative, car la conversation entre les héros est très importante. Leur peur exprime une grande déception ; par conséquence chaque personnage prend spontanément des distances pour se protéger. Nous rencontrons des passages où le dialogue se transforme en narration à la troisième personne ;

«-Mais il ne regarde personne, on dirait qu'il ne sait plus se servir de ses yeux. C'est pourquoi il me semble parfois moins heureux qu'on pourrait le croire... »

Remarquons la reprise des termes qui peut servir à enchainer les répliques classiques des personnages, la succession des questions et des réponses. La réponse à la question «pourquoi » ajoute deux raisons qui découvrent l'intrigue pour la jeune fille et aussi pour le lecteur. Dans leurs conversations, les personnages utilisent des questions verbalisées et des phrases elliptiques :

«- Ah ! monsieur. Vous me donneriez envie de mourir.

-Moi particulièrement, ou est-ce une façon de parler? -

[ $\quad \ldots]$

-parce qu'il y a aussi que je n'aimerais pas tellement,

Mademoiselle, avoir provoqué chez quelqu'un, ne serait-ce qu'une seule fois dans ma vie, une envie aussi violente de quelque chose.

-Je m'excuse, Monsieur.

(Les techniques dramatiques dans le Square ..)Dr. Ahmed Moawad Abd-Elhadi 
-Oh! mademoiselle, cela n'a aucune importance.

-Et je vous remercie aussi.

-Mais de quoi?

-Je ne sais pas, Monsieur, de votre amabilité. »

La diversité des dialogues est liée au domaine de l'énonciation définie comme une "mise en fonctionnement de la langue par un acte individuel d'utilisation » ${ }^{(24)}$ Observons que les dialogues sont inachevés, réduits à l'essentiel, lourds d'un sens qu'on pressent sans vraiment pouvoir le saisir, et que certains dialogues viennent quelquefois interrompre le récit du narrateur. Celui-ci à souligner les verbes introducteurs dans la citation :

«-Alors, cette femme vient à la cuisine, disiez-vous ?

-Oui, elle vient parfois. C'est le seul moment de la journée où elle vienne. Elle me demande toujours la même chose : comment ça va pour moi.

- Tout comme si cela pouvait aller pour vous différemment la veille du lendemain?

-Oui, tout comme. »

La plupart des interrogations se présentent dans le roman sous la forme du discours direct. Trouvons que la question est bornée aux conversations pendant les grandes scènes, dans lesquelles le personnage se trouve dans une situation d'énervement et d'agitation :

«-Oui, Mademoiselle. Je voyage. P. 11

-Je ne sais pas. P.15

-Mais, dans ce pays, il y a des gens malheureux, non ?

C'est vrai, oui. P. 35

-Combien de personnes, mademoiselle, si je peux me

permettre? p. 52

-A votre tour, que lui dites-vous, Mademoiselle ? p. 93

-Monsieur, ne parlons plus de moi. P 134

-Malheureux comment, Monsieur?

(Les techniques dramatiques dans le Square ..)Dr. Ahmed Moawad Abd-Elhadi 
-Puis cela s'est passé. P.134

-Oui, Puis cela s'est passé. P.135

-Et c'est ce que vous désirez aussi. P. 136

-Non, vous le ferez. P. 137

- A rien. J'aurais refusé, c'est tout. P. 139

- Peut-être que je me trompe, en effet. P. 140 »

Marguerite Duras se sert avec abondance du discours indirect libre. Un risque de confusion s'opère alors entre des questions appartenant à la forme dialogue, des questions ayant posées à l'oral entre les personnages et d'autres appartenant à la sous-conversation. Ainsi, si le roman dialogué "intègre des éléments formels du drame, il reste fondamentalement différent $d u$ drame » ${ }^{(26)}$. Un ouvrage à multiples interprétations : Certains privilégieront une portée réaliste et politique (Engagement communiste de Duras ), d'autres se glisseront entre les silences pour y déceler tous les ferments d'une quête amoureuse là où certains trouveront de quoi nourrir leur tourment existentiel et leur propre introspection.

C'est-ainsi que Le square est un texte théâtral qui déstabilise à première vue : entièrement constitué d'un seul dialogue entre une jeune fille et un vieil homme qui se viennent de se rencontrer, "il n'y a pas d'intrigue et le livre consiste en les diverses réflexions des deux protagonistes. » ${ }^{(27)} \mathrm{Et}$ pourtant, ce roman, adapté ensuite par Duras elle-même en pièce de théâtre, possède un véritable charme. En effet, si le roman dialogué « présente son énonciation en suppléant la désincarnation du littéraire par des effets illocutoires et pragmatiques qui motivent les enchaînements. » ${ }^{(28)}$, le drame joue son énonciation dans l'espace concret de la scène.

La courtoisie est aussi une qualité de ce dialogue. Les questions de l'un, parfois indiscrètes, trouvent une réponse franche, même si elle est intime. C'est là aussi volontairement 
exagéré par Marguerite Duras car ça lui permettait de rendre plus facile le lien qui se tisse entre eux, plus rapide aussi, en laissant de côté les règles implicites de sociabilisation. La rapidité dans la construction du dialogue est quelque chose d'important dans ce livre, tous les deux sont en état d'urgence, pressé de se rapprocher de l'autre. Ils sont à l'écoute de l'autre, du moindre geste. Ils discutent de leurs visions de la vie, de leurs problèmes, ouvertement, ils sentent implicitement que plus ils iront vite dans leurs relations, fit-elle sans suite, plus ils auront de chance de parvenir à leurs fins. Pour elle, un mariage avec lui. Pour lui, le renouveau de sa croyance en l'avenir.

\section{Les didascalies}

La didascalie réfère à la "indication scénique n'appartenant pas au texte même, dans une ouvre théâtrale. " ${ }^{(29)}$ C'est ainsi qu'il est intéressant de remarquer la souplesse du roman dialogué, qui intègre et transforme des éléments appartenant traditionnellement à d'autres genres littéraires et notamment au thêâtre. En examinant encore une fois des exemples révélateurs des possibilités de théâtralisation du roman dialogué, nous essayerons de voir en quoi il se distingue néanmoins du texte dramatique. La majorité des " romans dits dialogués que nous avons consultés place le dialogue sous la tutelle d'éléments paratextuels ou narratifs qui varient selon les romans. "(30). Nous trouvons dans ce roman un discours didactique, comme une pièce de théâtre, qui décrit les gestes, les regards, le comportement des personnages.

Une pièce de théâtre est écrite pour être vue et non pour être lue à la différence du roman qui offre de multiples façons. L'usage de l'italique, ainsi que la brièveté de l'information, n'est pas sans rappeler le fonctionnement des didascalies, impression renforcée par la suite avec l'emploi du nom de l'interlocuteur au début de chaque réplique. De la même manière, nous voyons que 
Le Square comporte plusieurs didascalies que l'on peut trouver dans une pièce de théâtre. Les didascalies selon Theuret ne sont pas «seulement perçues comme des messages successifs, mais comme des messages simultanés qui fonctionnent sur rythmes différents. » ${ }^{(31)}$ Dans le dialogue, c'est le personnage qui parle, tandis que, dans les didascalies, c'est le narrateur lui-même. Elles exigent un monde de lecture particulier.

Les didascalies dans le roman désignent quelques informations qui accompagnent le dialogue ou qui se trouvent au milieu de la conversation des personnages. Dans ce roman, nous pouvons voir comment les didascalies sont internes, dans lesquelles les personnages jouent un rôle. De la même manière, pouvons dire que tous les dialogues déjà traités contiennent des didascalies internes et externes. Il est nécessaire de mettre la lumière sur certaines didascalies dans le texte dialogué afin de relever cette dialectique qui organise le fonctionnement du texte, et les personnages à travers les rapports qui les unissent. Duras donne des indications concernant les gestes des personnages et traduisant le comportement des personnages à travers la didascalie qui désigne un geste sans parole. Ecoutons ce bref dialogue entre le vendeur et la jeune fille :

«-Je comprends, dit l'homme en souriant. Je n'en ai pas non
plus.
- Quelquefois cela parait curieux qu'il y en ait tant, et qu'on
n'en ait aucun à soi, vous ne trouvez pas?
-Sans doute, Mademoiselle, mais il y en a tellement déjà,
non? (32)

Dans un autre passage, voyons deux personnages qui partagent le même jeu par des didascalies dans lesquelles ils font des gestes lents pour qu'ils provoquent leur amitié de l'enfance. La jeune fille a été surprise par la présence de l'homme dans la rue, elle fait des mouvements pour l'atteindre : il se demande sur 
l'identité de la jeune fille qu'elle voit dans la rue. Ils échangent les idées et les regards suivants en silence :

«- N'empêche, Monsieur.

- Mais quand on les aime, quand ils vous plaisent beaucoup, est-ce que cela n'a pas moins d'importance?

- Ne pourrait-on pas dire aussi bien le contraire?

- Sans doute, Mademoiselle, oui, cela doit dépendre de son caractère. Et il me semble que certains peuvent se contenter de ceux qui sont déjà là. $\left[\begin{array}{ll}{[} & \ldots\end{array}\right]$

- Je vois, dit aimablement la jeune fille.

- Sauf en ce moment où je me repose, je voyage tout le temps.

- C'est un endroit bien indiqué, les squares, pour se reposer, en effet, surtout en cette saison. J'aime bien les squares, moi aussi ; être dehors.

Dans ce passage, la didascalie est très importante et les gestes complètent le sens de la scène. L'on voit comment les deux amis d'autrefois se reconnaissent et comment ils se retrouvent dans le même square. Remarquant que la conversation entre les personnages a pour eux une triangulation initiale et finale. Cette triangulation se déroule dans le jardin ouvert où se trouve un couple qui ne s'aime pas et un enfant :

« L'enfant regarda cet homme qui lui souriait, complètement

indifférent. Puis il retourna vers le sable. La jeune fille le suivit des yeux.

«Il s'appelle Jacques, dit-elle.

-Jacques », répéta l'homme.

Mais il ne pensait pas à l'enfant. »

Ce procédé typiquement théâtral permet d'identifier les locuteurs, mais aussi de rendre explicite le nombre de participants. Ainsi, le lecteur suit réplique après réplique le dialogue entre les trois personnages, échangeant sur leurs 
aventures amoureuses. Cependant, le dialogue de Jacques nous décrit le rôle de la jeune fille en didascalies :

«Je l'insulte, et sans jamais lui donner la moindre

explication. Pourquoi, dites-moi, Monsieur?"

En ce sens, chaque scène dialogale fonctionne comme une des valeurs partagées faisant partie de l'interdiscours ${ }^{(36)}$ soit romanesque ou théâtrale. Aussi bien pour le roman que pour le théâtre, les didascalies sont-elles associées à des types d'activités narratives précises, nous pouvons bien l'observer dans le passage suivant où la jeune fille partage le même jeu par des didascalies :

«Elle sautille, et lui se traine, ce qui fait qu'ensemble...Ah...je ne saurais vous les décrire. Ce n'est pas leur faute, me direz-vous... » 37 )

C'est une ouverture sur le monde extérieur, sur l'autre. Le sautillement assure le libre passage du sujet à l'objet, de l'intérieur à l'extérieur, du réel au mental qui permettra l'accomplissement de la destinée de la fille par le changement. Remarquons que les didascalies accompagnent évidemment le dialogue. Elles sont destinées à orienter et faciliter l'élaboration narrative. Elles sont aussi des ajouts explicatifs qui permettent au lecteur de savoir beaucoup d'informations et de renseignements sur les personnages et la structure du roman. Ils sont également un trait d'union entre le dialogue et ce que décrit le narrateur.

\section{Les gestes :}

Il est évident que ce roman se réduit aux mouvements des corps de personnages. Depuis son abandon par l'enfant et pendant le roman l'homme semble exister comme un automate. Toujours en mouvement, il se cogne, se bascule, court, vole selon un rythme accéléré. Duras ne lui donne l'occasion de reprendre son souffle et la plonge dans des situations folles à cause de son mouvement dans la ville. A ce propos, H. Bergson dit que «les attitudes, gestes et mouvements du corps humain risibles dans 
l'exacte mesure où ce corps nous fait penser à une simple mécanique. ${ }^{(38)}$ Cela nous entraine à un aspect comique par ses gestes automatiques comme nous remarquons le geste de la jeune fille répondant à l'interrogation de l'homme sur l'enfant :

« Il est gentil » dit l'homme.

La jeune fille secoua la tête en signe de dénégation.

«Ce n'est pas le mien », dit-elle. »

Revenue à l'homme, il se remet à un mouvement continue, à travers ses longues errances dans les villes. Comme le mendiant, il marche, interminablement, des après-midis entiers. Cette situation lui permet de s'absenter en dehors pour savoir la vérité :

"La jeune fille les essuya dans un geste léger et sûr.

L'homme sourit à l'enfant. "Si je le disais, fit-il, c'était simplement pour le remarquer, pour rien d'autre que pour le remarquer $(40)$

L'homme suit les couples à la venue du matin, puis il les poursuit dans les rues afin de les surprendre. De même, la jeune fille à son tour le comportement de Jacques et se met à la suivre sans être vu. Au centre du triangle formé par l'homme, la jeune fille et Jacques, les places s'échangent, jusqu'à l'éviction de la jeune fille, elle-même devant le ravissement opéré par Jacques de son amant. Ce jeu d'échange se matérialise par le rôle de l'espace à travers le champ de seigle. Et l'on voit la femme avec un regard très dur sur sa condition. Elle estime que son métier est difficile, elle se pense humble, abandonné. Elle estime que sa condition est moindre que celle de l'homme, qui ne la corrige pas. Il faut dire que lui croit encore pouvoir faire quelque chose dans le futur, ce que ne croit pas la femme.

Certes, les dialogues à l'aide des gestes simulent dans le roman une forme d'interactions verbales assumées par les personnages. Dans un autre passage, nous voyons des personnages partager le même jeu. Le narrateur relève les indices 
visuels, kinésiques et qu'exprime la disposition des corps les uns par les autres. Le geste est ici représenté par les caresses et les regards. La jeune fille est voyeuse. La caresse se déploie en parallèle, tandis que Jacques observe leurs gestes.

«-Pourquoi cette colère contre ce chien, Monsieur ? Malgré tout ce qu'il mangeait, lui c'était son seul ami. On ne croit pas être méchant et pourtant, voyez !

-Mademoiselle, cela ne devrait pas exister. Or, du moment que cela existe quand même, nous ne pouvons pas éviter, à notre tour, de faire des choses que nous ne devrions pas faire. C'est inévitable, absolument inévitable. »

Il est à remarquer que les déambulations de l'homme à travers les espaces ouverts des rues de sa ville natale évoquent la libération progressive d'une personne que la perte de l'être aimé avait enfermée dans un comportement de quasi-robot.

La jeune fille suit Jacques et l'homme à la place où le narrateur voit le point de départ du destin qui aboutit l'homme au bal fatal. La jeune fille retourne vers le passé, elle veut recréer une atmosphère propice.

«-Monsieur, je suis bonne à tout faire et j'ai de l'espoir. Il ne faut pas parler comme ça.

-Je m'excuse, Mademoiselle, je m'explique mal. Vous, vous changerez; moi, je ne le crois pas, je ne le crois pas, je ne le crois plus. Et, que voulez-vous, il n'y a rien à faire, même si je ne l'ai pas tout à fait voulu, je ne peux pas oublier ce voyageur de commerce que je suis. A vingt ans je mettais des shorts blancs et je jouais au tennis. C'est ainsi que les choses commencent, n'importe comment. On ne le sait pas assez.

Cela nous rappelle un autre aspect des gestes : c'est le pantomime qui consiste à faire des gestes sans que les personnages recourent au langage pour exprimer leurs sentiments. 
Ils doivent être capables de les exécuter minutieusement. Il existe des scènes montrant des pantomimes d'un effet irrésistible. C'est ainsi que, nous rencontrons quelques indications complétées par les mouvements des corps. Ça nous rappelle les mimiques. Tous ces verbes indiquent les gestes des personnages : avancer, reculer, se lever, retourner et ....

"-Oui, Monsieur, sans doute, mais pourquoi la lâcheté a-t-

elle tant d'attrait et si peu le courage, vous ne trouvez pas?

Toujours la lâcheté, Mademoiselle, mais c'est si facile, si

vous saviez

Le petit garçon tira la main de la jeune fille.

" Je suis fatigué », déclara-t-il encore.

L'homme leva les yeux et parut s'inquiéter un peu.

«Aurez-vous des observations, Mademoiselle?»

C'est ainsi que nous pouvons distinguer un bal énigmatique tissé d'approches des corps ; un bal dont les tensions les sursauts, les respirations forment en définitive l'essentiel le square. Marguerite Duras nous a offert un roman visuel avant tout. Le référent «bal » peut être identifié' il s'agit d'être deviné par le récepteur extralinguistique ou extratextuel tentant de combler des lacunes en tenant compte du contenu sémantique

\section{Le monologue}

Selon Benveniste le monologue «doit être posé, malgré l'apparence, comme variété de dialogue, structure fondamentale. » (44) Dans le théâtre, le monologue est un discours d'un personnage qui est ou qui se croit seul sur scène. Sa fonction est de connaitre un personnage de l'intérieur. Les dramaturges y ont recours sans cesse d'en atténuer le caractère conventionnel, et ils s'en servent souvent à un moment où l'action plonge le héros dans un tumulte émotif et passionnel. ${ }^{(45)}$ Dans le Square, nous rencontrons des situations identiques de monologues théâtraux et bien qu'on parle de roman. Les monologues qu'utilisent les 
personnages symbolisent fréquemment leur impossibilité à se comprendre ou à communiquer avec autrui. Le récit de l'enfant (Jacques) comporte de nombreux monologue intérieur surtout après chaque dialogue. Il ne faut pas étonner De l'entendre dire, quand l'homme commence à se souvenir des lieux et s'approchant de la gare de sa ville natale :

«-Mais je ne veux pas dire, à mon tour, que je suis
malheureux ou triste quand je dis que parfois j'éprouve un
besoin de parler si vif que je m'adresse à des enfants. Non,
ce n'est pas ça puisque j'ai quand même un peu choisi la vie
que j'ai là, ou alors il faudrait que je sois fou pour avoir
choisi mon malheur. » (46)

L'orientation vers le monologue est dans ce contexte un phénomène propre dans la narration, car on peut en rencontre un autre, il s'agit d' « étranger » qui ne peut pas éviter à travers la narration les actions vives et intenses avec la parole d'autrui. Le narrateur de ce récit vit les événements. Il utilise des nombreux monologues autonomes ou remémoratifs qui valorisent l'importance de l'homme et la fascination qu'il subit de la jeune femme se révèle depuis ses premières paroles. Il parle ainsi de son propre enchantement :

\footnotetext{
«Encore une fois, quand je parlais de cette femme et des gens qui évitent d'être tout à fait heureux, je ne voulais pas dire par là qu'il ne fallait pas pour autant ne pas suivre leurs exemples, ne pas essayer à son tour, et échouer à son tour. » (47)
}

Comme on peut le voir, l'homme montre d'une part un éblouissement que reflète l'importance des regards, il traduit d'autre part son impossibilité d'annoncer son amour pour la jeune fille. Il souffre silencieusement. Donc il y a une correspondance entre l'écriture et l'événement. De là nait une coïncidence entre le 
temps de la narration et celui de l'histoire comme on voit dans l'extrait suivant :

«Parfois je ne sais plus. J'avais seize ans quand ça a commencé. Je n'y ai pas pris garde au début et puis, maintenant, voilà que j'ai vingt et un ans et que rien ne m'est arrivé, rien, et voilà qu'il y a en supplément cette vieille grand-mère qui n'en finira pas de mourir, cependant que personne encore ne m'a demandé d'être sa femme.»

Cet incipit monologué donne un ton au roman relatant un fragment court de la vie humaine à travers monologuiste. La narration monologuée plonge ensuite le lecteur dans un monde particulier en dressant un des brefs portraits. C'est ainsi que la mise en scène du temps reste éloignée de la réalité. Le narrateur a procédé une sorte d'accélération. On peut dire qu'il existe deux segments opposés :

«Parfois je me demande si je ne rêve pas, si je n'invente pas

tant de difficultés. »

Il se soumet ainsi à ses hésitations et ses balbutiements d'homme-amant. D'autre part, ce monologue ne peut pas éviter selon Bakhtine - « une [inter]action vive et intense » ${ }^{(50)}$ avec le discours du narrateur. On rencontre aussi certains monologues dans lesquels le narrateur nous communique ses interrogations du moment : nombreuses questions se trouvent sans réponse :

" - Mais quand on les aime, quand ils vous plaisent beaucoup, est-ce que cela n'a pas moins d'importance?

contraire ?»

-Ne pourrait-on pas dire aussi bien le (51)

Par rapport à tous ces citations monologuées, nous remarquerons que la notion de monologue est complexe, car il peut être placé en plusieurs niveaux fonctionnels (littéraire, communicatif et lectorat). Comme on l'a vu, les nombreux monologues utilisés dans ce roman font penser à des mises en 
scènes théâtrales. Parfois, l'ennui nait de la monotonie de certains monologues. Il y a des paragraphes qui ressemblent à des monologues qui se représentent sur scène. Après sa première tentative de retour au Square, Jacques accomplit un voyage avec la jeune fille et il fonde son autorité narrative.

«- Cela n'a pas d'importance, allez. Non, si je vais
quelquefois dans les squares, c'est quand je suis resté
quelques jours sans parler, vous voyez, sans bavarder, quoi,
quand je n'ai pas eu d'autre occasion de le faire qu'avec des
gens qui achètent ma marchandise, et que ces gens sont
pressés ou tellement méfiants que je ne peux arriver à leur dire un mot en dehors de ceux pour vanter mes cotons.»

Duras exprime ici un paysage plein des sens grâce au mélange des eaux, une métaphorisation évidente de l'acte du silence et de la jouissance. Le paysage confus désigne ici dans le même temps l'union amoureuse et la rencontre du présent avec le passé. De même, l'homme emploie des monologues intérieurs concernant son futur amant; on peut affirmer que cet homme ne veut pas oublier son enfance qui l'a abandonnée. Après son retour au Square, au moment où il surprend une jeune fille inconnue sortant du cinéma, il s'interroge sur son sujet. Cette structure fait écho aux monologues dans une pièce de théâtre. Remarquons que les formes interrogatives sont très bizarres Ainsi la dernière phrase affiche-t-elle un mode exclamatif. Nous pouvons imaginer le ton de l'homme qui suggère un questionnement.

\section{Conclusion}

Pour conclure, nous pouvons dire que Marguerite Duras a écrit ce roman dialogué qui se présente bel et bien comme une fiction de l'oralité puisque la parole est partiellement prise en charge par les personnages, sans recours systématique à un narrateur. Le Square a présenté à cet égard, des affinités avec l'univers théâtral. 
L'histoire de ce roman en somme l'histoire de l'aliénation mentale d'une femme frappée dans son équilibre nerveux et qui ne peut plus vivre qu'en dehors d'elle-même.... Le narrateur, cherchant de comprendre le vendeur, cherche en même temps les raisons qui l'attirent vert cette jeune femme dont la douce folie est le seul instrument de séduction.

Dans ce roman reviennent des indications qui désignent l'histoire de la jeune fille sous le signe du spectaculaire : comme le spectacle ravissant de bal du vendeur; la deuxième rencontre entre le vendeur, Jacques, et Jeune fille se fait ainsi à l'occasion d'une tournée théâtrale; la folie de cette fille la rend faire des tournées, ce qui nous rappellera l'univers théâtral.

Particularité totalement assumée par Marguerite Duras que de celle de créer un dialogue complètement décalé par rapport à la condition des personnages. Non seulement à une forme de dialectique philosophique, mais la forme langagière n'appartient à aucun registre codé. On se surprend à penser à Camus ou du Beckett avec quelques tonalités flirtant de près avec l'absurde. L'on notera aussi l'étrangeté d'un langage curieusement dépersonnalisé pour mieux parler de l'intime et de sa condition. Comme on l'a vu, toutes ces techniques-les scène fantasmatiques, le dialogue, les didascalies, les gestes et les monologue analysésfont le square une vraie pièce de théâtre. 
1 - Luthi. J.J, La littéraire d'expression française en Égypte (1798- 1998), Édition : L'Harmattan, 2000, Nouvelle édition remaniée, p. 16

2 - Duras (Margueritte), Le square, Galimard, Paris, 1983.

3 - Ariette Armel, Margueritte Duras et l'autobiographie, Le Castor Astral square, Paris, 1990, p. 117

4 - Argaud., (Évelyne) «Sensibiliser à l'altérité par le texte francophone », dans la revue : Le Français dans le Monde, revue de la fédération

internationale de professeurs de français, Mai- Juin, Édition : Didier, 2004, No: 333 . p. 34

5 - Le Grand Robert 2005, version électronique 2.0

${ }^{6}$ - https://www.babelio.com/livres/Duras-Le-Square/25528/critiques?note=5

7 - Le square, op.cit., p.p.19, 20

8 - cf. Borgomano (Madeleine), Commente Le Ravissement de Lol V. Stein, Gallimard, Paris, 1997.

9 - Radiguet (Raymond), Le bal du Comte d'Orgel, Gallimard, Paris, 1938.

P. 64

${ }^{10}$ - Le square, op.cit., p.p. 22,23

${ }^{11}$ - https://www.babelio.com/livres/Duras-Le-Square/25528/critiques?note=5

12 - Le square, op.cit., p. 47

13 - Cousseau (Anne), Une dramaturgie de la mémoire, in Lectures de Duras, P.U de Rennes. Paris. 2005, p. 34

${ }^{14}$ - Le square, op.cit.

15 - https://www.babelio.com/livres/Duras-Le-Square/25528/critiques?note=5

16 -Boblet, (Marie-Hélène), « Topique romanesque et déconstruction narrative dans Mercier et Camier », Marius Buning (dir.), Three dialogues revisited/Trois dialogues revisités, Samuel Beckett revisité, vol. 13, Rodopi, 2003, p.13

17 - Corvin (Michel), Dictionnaire encyclopédique du théâtre, Bordas, Paris, 1991.

18 - Le square, op.cit. p. 9

19 - Ibid., p. 19

20 - Ibid., p.54

${ }^{21}$ - Ibid., p. 15

22 - Ibid. p. 95

${ }^{23}$ - Ibid. pp.76, 77

(Les techniques dramatiques dans le Square ..)Dr. Ahmed Moawad Abd-Elhadi 
${ }^{24}$ - Benveniste (Emile), Problèmes de linguistique générale, Gallimard, Paris, 1974, p.80

25 - Le square, op.cit. p. 100

${ }^{26}$ - Galophe (Marie), La force discursive des passions dans le roman dialogué : Éole et les âmes fortes, Thèse de doctorat en lettres françaises Département de français Faculté des études supérieures et postdoctorales Université d'Ottawa (C) Canada, 2013. p. 203

${ }^{27}$ - https://www.babelio.com/livres/Duras-Le-Square/25528/critiques?note $=5$

${ }^{28}$-Boblet (Marie-Hélène), op.cit., p.69

${ }^{29}$ - Le Grand Robert 2005, version électronique 2.0, op. cit

${ }^{30}$ - Galophe (Marie), op. cit, p. 200

31 - Theuret (F. Rullier), Le texte de théatre, Hachette, Paris, 2003, p. 11.

32 - Le square, op.cit. p.10

${ }^{33}$ - Ibid. p. 11

${ }^{34}$ - Ibid. p. 82

35 - Ibid. p. 93

${ }^{36}$ - cf. Maingueneau (Dominique), Les termes clés de l'analyse de discours, Seuil, Paris, 1996, p 101

37 - Ibid. p. 126

38 - Herni (Bergson). Le rire, essai sur la signification du comique, P.U.F, Paris,1961, pp.22,23

${ }^{39}$ - Le square, op.cit. p. 10

${ }^{40}$ - Ibid. pp. 81,82

${ }^{41}$ - Ibid. pp.108,109.

${ }^{42}$ - Ibid. pp. 122.

${ }^{43}$ - Ibid. pp. 152.

${ }^{44}$ - Benveniste (Emile), op.cit, p.85

${ }^{45}$ - Alain (Couprie), Le théâtre, Armant Colin, Paris, 2005, p.15.

46 - Le square, op.cit. p.90

${ }^{47}$ - Ibid. p. 101

48 - Ibid. p. 119

${ }^{49}$ - Ibid. p. 119

50 - Bakhtine (Mikhail), Esthétique et théorie du roman, Gallimard, Paris, 1978, p. 102

${ }^{51}$ - - Le square, op.cit., p. 11

52 - Ibid. p. 90 


\section{BIBLIOGRAPHIE}

\section{Corpus de l'étude}

- Duras (Margueritte), Le square, Galimard, Paris, 1983

\section{Ouvrages de critique}

- Alain (Couprie), Le théâtre, Armant Colin, Paris, 2005

- Ariette (Armel), Margueritte Duras et l'autobiographie, Le Castor Astral square, Paris, 1990

- Bakhtine (Mikhail), Esthétique et théorie du roman, Gallimard, Paris, 1978

- Barthes (Roland), Le plaisir du texte, Seuil, Paris, 1973

- Bessiere (Jean), Le roman contemporain ou la problématicité du monde, P.U.F, Paris, 2010

- Boblet (Marie-Hélène), « Topique romanesque et déconstruction narrative dans Mercier et Camier », Marius Buning (dir.), Three dialogues revisited/Trois dialogues revisités, Samuel Beckett revisité, vol. 13, Rodopi, 2003

- Borgomano (Madeleine), Commente Le Ravissement de Lol V. Stein, Gallimard, Paris, 1997

- Charles (Grivel), Production de l'intérêt romanesque - un état du texte (1870-1880), un essai de constitutions de sa théorie, Mouton, The Hague, Paris, 1973

- Combe (D), Les genres littéraires, Édition Hachette, Paris, 1992

- Corvin (Michel), Dictionnaire encyclopédique du théâtre, Bordas, Paris, 1991

- Cousseau (Anne), "Une dramaturgie de la mémoire », in Lectures de Duras, P.U de Rennes. Paris. 2005

- Maingueneau (Dominique), Les termes clés de l'analyse de discours, Seuil, Paris, 1996

- Dubois (Jean), Grammaire structurale du français; nom et pronom, 1965, Librairie Larousse

- Genette (Gérard), Seuils, Seuil, Paris, 1987 
- Grall (Catherine), « Rhétorique, narratologie et sciences cognitives : quel statut pour le narrateur ? », Jean Bessière (dir.), Littérature, représentation, fiction, Honoré Champion, Paris, 2007

- Hamon (Philippe), Pour un statut sémiologique du personne, in Poétique du récit, col. Points, 1977

- Hamon (Philippe), Le Roman des théories aux analyses, Texte et Idéologie, Ed : PUF, Paris, 1984

- Herni (Bergson). Le rire, essai sur la signification du comique, P.U.F, Paris, 1961

- Lüthi. (J.J), La littéraire d'expression française en Égypte (1798- 1998), Édition, L'Harmattan, Paris, 2000, Nouvelle édition remaniée.

- Radiguet (Raymond), Le bal du Comte d'Orgel, Gallimard, Paris, 1938.

- Theuret (F. Rullier), Le texte de théâtre, Hachette, Paris, 2003.

- Ricoeur (Paul), Le Temps et récit II, Seuil, Paris, 1984

- Rullier (Theuret. Françoise), Approche du roman, Ed: Hachette Livre, Paris, 2001

- Todorov (Tzvetan), Introduction à la littérature poétique, Seuil, (Coll. «Points/Essais), Paris, 1976

- Wellek ??? \& Warren (Austin), La théorie littéraire, Seuil, Paris, 1971

\section{Thèses}

- Galophe (Marie), La force discursive des passions dans le roman dialogué : Éole et les âmes fortes-Thèse de doctorat en lettres françaises Département de français Faculté des études supérieures et postdoctorales Université d'Ottawa (C) Canada, 2013

- Nabil (Oussama), Thèse de doctorat, Le réel et l'imaginaire chez Tahar Ben Jelloun, Université de L'Azhar- faculté de langues et de traduction, Le Caire 1993 


\section{Revues}

- Argaud. (Évelyne), «Sensibiliser à l'altérité par le texte francophone», dans la revue: Le Français dans le Monde, revue de la fédération internationale de professeurs de français, Mai- Juin, Édition : Didier, 2004, No : 333

- Arnaud (Jacqueline), «Khaire Eddine le sudiste», in revue littéraire, n 21 octobre 1980

- Guingamp, (Pierre). Hafez el Assad et le Parti Baath en Syrie (Comprendre le Moyen-Orient). Éditions L'Harmattan, 1996. (ISBN 2738446787 et 9782738446787 ).

\section{Internet}

-https://www.babelio.com/livres/Duras-Le-Square/25528/critiques?

$\underline{\text { note }=5}$

http://fr.wikipedia.org/wiki/Jacques_Becker

www.almasryalyoum.com (consulté le 4 décembre 2010)

-https://www.babelio.com/livres/Duras-Le-

Square/25528/critiques?note $=4$

http://fr.wikipedia.org/wiki/Hafez_el-Assad

http://fr.wikipedia.org/wiki/Jean-Paul_Sartre

www.almasryalyoum.com (consulté le 4 décembre

http://www.woodyguthrie.org/Lyrics/Ingrid_Bergman.htm [archive]، 


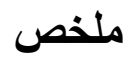

يعالج البحث مظاهر الرواية المسرحية في رائعة مارجربت دوراس الميدان.

إنها حقا رواية مسرحية بامتياز ، حيث تجسدت فيها الحوارات وأحاديث النفس وكذلك

سينوغرافيا المكان. فما أن توجد هذه المكونات مجتمعة في رواية، حتى تصح أن يطلق عليها رواية مسرحية. ارتكز بحثنا على التقنيات التي تميز كتابات دوراس في روايتها الميدان، وفي الوقت نفسه، عملنا على استخراج ملامح المسرحة في الرواية عبر تسليط الضوء على الحوار، المونولوج، وكذلك الإشارات المسرحية تلك التي شكلت في نهاية المطاف المساحة الاكبر في الرواية. 